\title{
Non-fluorinated non-solvating cosolvent enabling superior performance of lithium metal anode battery
}

Jun yeob Moon

Seoul National University

Dong Ok Kim

Seoul National University

Lieven Bekaert

Vrije Universiteit Brussel https://orcid.org/0000-0003-1776-1888

\section{Munsoo Song}

Seoul National University

Jinkyu Chung

Seoul National University

Danwon Lee

Seoul National University

Annick Hubin

Vrjie Universiteit Brussel

Jongwoo Lim ( $\square$ jwlim@snu.ac.kr)

Seoul National University https://orcid.org/0000-0002-3897-7488

\section{Article}

Keywords: Lithium-ion solvation, lithium metal anode, fluorinated non-solvating cosolvents, NFNSC

Posted Date: November 9th, 2021

DOI: https://doi.org/10.21203/rs.3.rs-971099/v1

License: (c) (1) This work is licensed under a Creative Commons Attribution 4.0 International License. Read Full License

Version of Record: A version of this preprint was published at Nature Communications on August 4th, 2022. See the published version at https://doi.org/10.1038/s41467-022-32192-5. 


\section{Non-fluorinated non-solvating cosolvent enabling superior}

\section{2 performance of lithium metal anode battery}

3 Junyeob Moon ${ }^{1, \dagger}$, Dong Ok Kim ${ }^{1,2, \dagger}$, Lieven Bekaert ${ }^{3}$, Munsoo Song ${ }^{1}$, Jinkyu Chung ${ }^{1}$, Danwon

$4 \quad$ Lee $^{1}$, Annick Hubin ${ }^{3, *}$, Jongwoo Lim ${ }^{1, *}$

$5{ }^{1}$ Department of Chemistry, College of Science, Seoul National University, Seoul, 08826,

6 Republic of Korea

$7 \quad{ }^{2}$ Department of Chemistry and Biochemistry, Swarthmore College, Swarthmore, PA 19081,

8 United States of America

$9{ }^{3}$ Vrjie Universiteit Brussel, Research Group Electrochemical and Surface Engineering,

10 Pleinlaan 2, 1050, Brussels, Belgium

$11 \dagger$ These authors contributed equally: Junyeob Moon, Dong Ok Kim

$12{ }^{*}$ Corresponding authors, e-mail: annick.hubin@vub.be, jwlim@snu.ac.kr 


\section{Abstract}

16 Lithium-ion solvation governs the performance of lithium metal anode (LMA) by tuning its

17 interfacial stability. Solvation degree is modulated by adopting fluorinated non-solvating cosolvents (FNSC) to induce anion-rich solvation structure which is beneficial in constructing mechanically stable interface to suppress lithium dendrite. However, FNSC exhibits low cathodic stability owing to their low lowest unoccupied molecular orbital (LUMO) level, aggravating long-term cycling of LMA. We establish that spectroscopically measured Lewis basicity and polarity are critical parameters for designing optimal non-solvating cosolvents. Non-fluorinated non-solvating cosolvents (NFNSC) proposed by our design rule (i.e. anisole, ethoxybenzene and furan) delivered $99.0 \%$ coulombic efficiency over 1400 cycles. In these molecules, the aromatic ring delocalizes oxygen electron pairs and lowers solvation capability, confirmed by electrochemical cycling, Raman spectroscopy, and DFT binding energy calculation. Finally, the quantification of remaining NFNSC in the electrolytes using nuclear magnetic resonance spectroscopy proves their reductive stability for extended cycles. 


\section{Introduction}

31 Cosolvents are widely developed for application in a lithium-ion battery (LIB) electrolyte to 32 enhance anion solvation ${ }^{1,2}$, flame retardancy $y^{3,4}$, lithium-ion migration ${ }^{5}$, and high-voltage stability ${ }^{6,7}$. An increase in the demand for high-energy density LIB requires the development of LMA owing to the remarkably high specific energy $y^{8,9}$. However, the dendritic growth of lithium metal, which leads to the failure of the battery, remains problematic ${ }^{10}$. Mechanically strong interphases are reportedly effective in preventing dendrite formation ${ }^{11}$. Recent studies have demonstrated that the addition of a non-solvating cosolvent to generate localized high concentration electrolyte (LHCE) can induce compact lithium-ion-anion solvation pairs that participate in high-strength inorganic SEI generation ${ }^{1,2} 12,13$.

Thus far, design strategies for ideal non-solvating cosolvents are largely unexplored and thus limited to only fluorinated non-solvating cosolvents (FNSCs) ${ }^{14-16}$. The negative inductive effect of fluorine withdraws electrons near the adjacent oxygen within the solvent molecules, which lowers lithium solubility ${ }^{17}$. Further, this decreases cathodic stability, and it results in a facile decomposition to LiF-rich $\mathrm{SEI}^{18-20}$. It has been largely overlooked that the accelerated decomposition to SEI consumes cosolvents, and this leads to electrolyte dry-up and battery failure ${ }^{21-24}$. Further, the high cost and hazardous environmental effect of FNSC necessitate the use of non-fluorinated compounds as non-solvating cosolvents ${ }^{15,25,26}$.

In this study, we present the design rule for ideal non-solvating cosolvents, which is justified by the superior performance of non-fluorinated non-solvating cosolvents (NFNSC) that cycle over 350 cycles ( $99.0 \%$, ethoxybenzene), 500 cycles (98.5\%, anisole (AN)), and 1300 cycles 
to identify the ideal physicochemical properties of non-solvating cosolvents. The resonating

\section{Results}

\section{Results} salt cost. electron pairs of an oxygen atom decreases the basicity of the NFNSC, thereby realizing desirable non-solvating characteristics while maintaining good miscibility, superior cathodic stability, and low price.

\section{Physicochemical properties of NFNSC and design strategy}

A non-solvating cosolvent, also known as a diluent, maintains the beneficial highconcentration electrolyte (HCE) solvation structure while lowering viscosity and production cost so that it can be applied to conventional cell geometries ${ }^{27,28}$. An $\mathrm{HCE}$ with $5 \mathrm{M}$ or a higher salt concentration renders the inorganic-rich SEI formation with a higher mechanical stability and stabilizes the LMA interface because of the lithium-ion-anion paired solvation structure. Contact ion pair (CIP) and aggregate (AGG) solvation geometries are created because there is no sufficient solvent molecule to solvate lithium-ions. However, the HCE cannot be considered for practical applications because of its high viscosity and expensive

The addition of non-solvating cosolvents that enable the LHCE structure is a considerably promising strategy because it can effectively lower the viscosity and cost of the HCE. The design of ideal non-solvating cosolvents remains challenging because multiple components need to be considered. Non-solvating cosolvents must not coordinate lithium-ions and be inert to LMA for preserving the local solvation shell of HCE while being miscible with the solvating solvent ${ }^{29}$. Conventionally, physicochemical parameters such as dielectric constant, 
74 dipole moment, and calculated binding energy have been considered for predicting solvation 75 properties and miscibility with other solvents ${ }^{14,16,30,31}$. However, the behaviour of a cosolvent 76 mixed with multiple components in the electrolyte deviates from that of the pure form ${ }^{32}$. The 77 binding energy of the solvent-lithium-ion pair calculated by density functional theory (DFT) 78 is limited to local interactions, and it is less accurate at predicting the complex solvation 79 energetics in real electrolytes (Figure 1c-d, inset) ${ }^{33}$. The dipole moment and dielectric 80 constant, which only capture the polarity properties of the pure component, fail to represent 81 either the solvation ability or miscibility with the solvating solvent and concentrated salts in 82 the ensemble (Figure S1c-d $)^{31,34}$. 
a
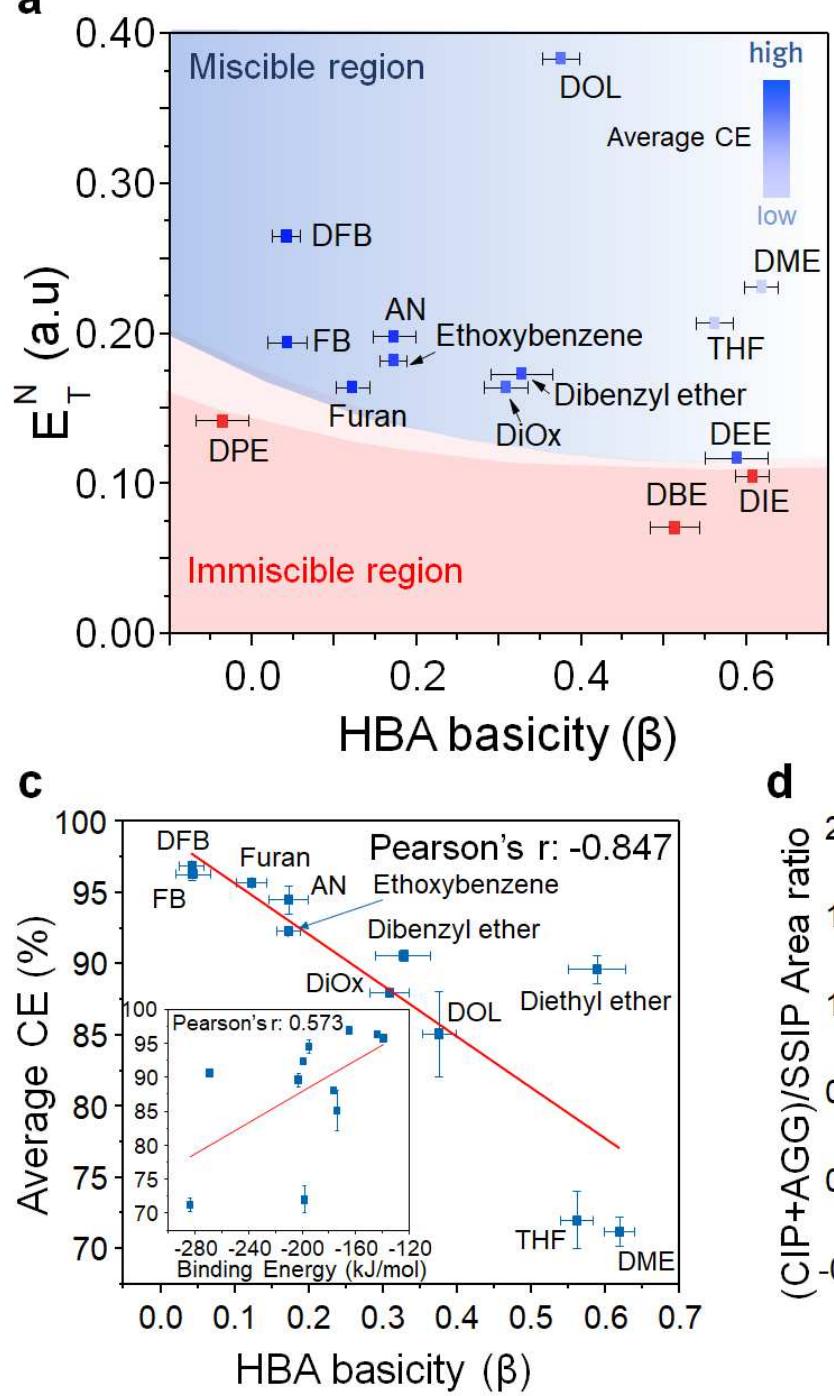

e

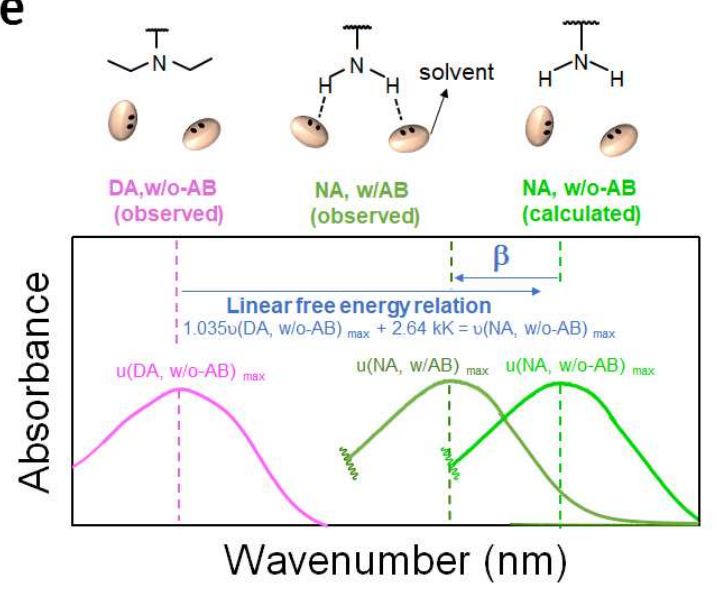

$f$ b

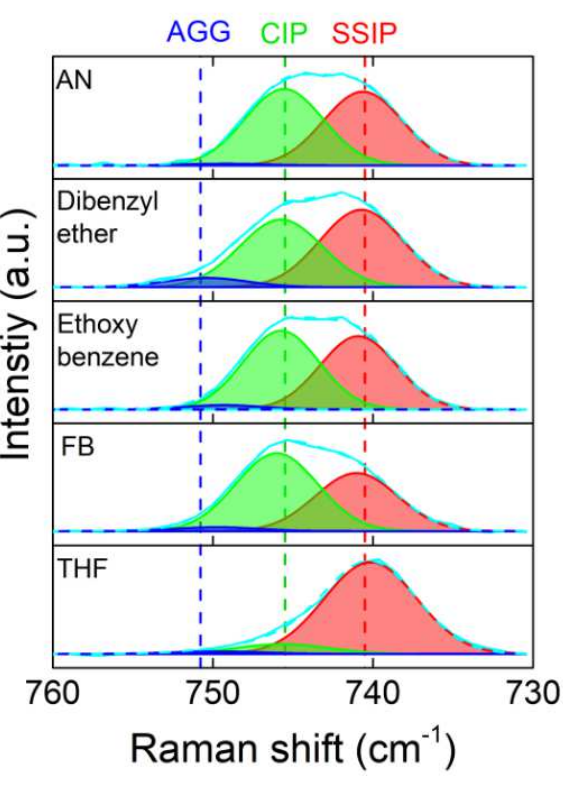

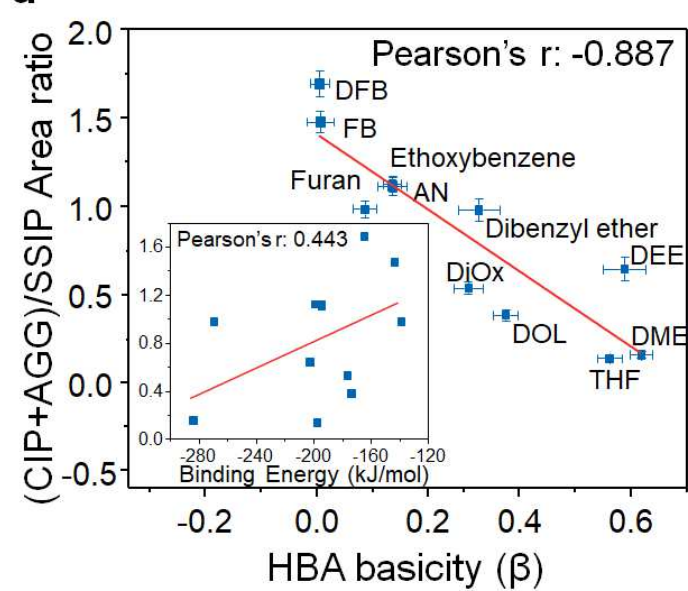

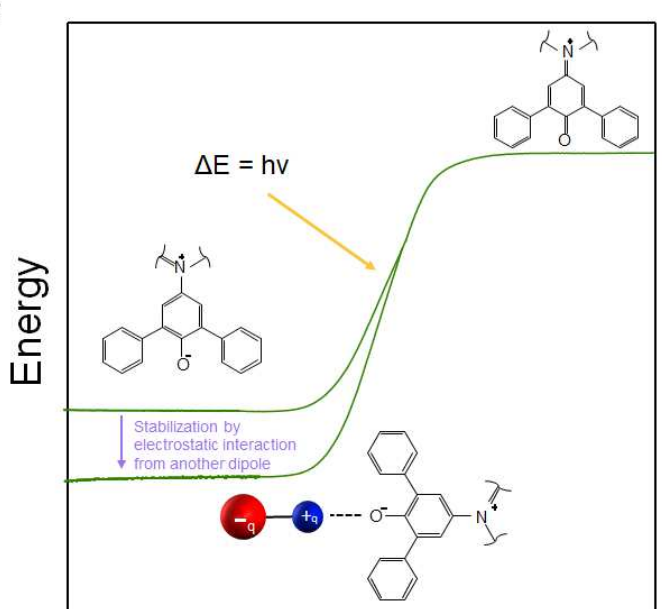

Figure 1. (a) HBA basicity vs. $E_{T}^{N}$ (normalized transition energy) two-dimensional plot of each solvent. (b) Raman peak deconvolution for TFSI ${ }^{-}$shift of electrolytes containing 
different cosolvents (EC/DEC:cosolvent $=1: 2$ ). (c) Correlation between average CE and HBA basicity. The average CE was obtained using an Aurbach method. The inset demonstrates the correlation between the average CE and binding energy. (d) Correlation between the Raman deconvolution result and HBA basicity. The inset demonstrates the correlation between the Raman deconvolution result and binding energy. (e) Schematic of HBA measurement using two different dyes. (f) Schematic of $E_{T}^{N}$ (normalized transition energy) measurement.

For example, the dielectric constant and dipole moment of tetrahydrofuran are 7.6 and $1.63 \mathrm{D}$, respectively; the values of 1,2-difluorobenzene are 14.26 and $2.53 \mathrm{D}$, respectively. Despite the higher values, the solvating property of 1,2-difluorobenzene is lower than that of tetrafydrofuran ${ }^{14,35}$. Thus, a new paradigm of solvent descriptors that considers mutual interaction with other components in the electrolyte should be established for designing newclass cosolvents ${ }^{34,36,37}$.

We propose experimental methods to characterize lithium-ion solvation ability and miscibility with other polar species in electrolytes spectroscopically. It is imperative to measure the stabilization energy of a lithium-ion in a specific solvation structure to assess the lithium-ion solvation ability. In order to mimic the phenomenon, we utilized dyes that form solvation structures with a solvent of interest and enable the measurement of the degree of stabilization. One dye contains a primary amine group $\left(-\mathrm{NH}_{2}\right)$ that forms a hydrogen bond with the surrounding solvent molecules while the other one, with the tertiary amine group ($\left.\mathrm{N}\left(\mathrm{CH}_{2} \mathrm{CH}_{3}\right)_{2}\right)$, does not form the hydrogen bond. The difference between the absorption energies of the two dyes represents their stabilization energies when solvated in a solvent through hydrogen bonding. The solvation around the hydrogen of the primary amine group may be close to that around the lithium-ion. This analogy thus mirrors the stabilization energy of a solvated lithium-ion in a specific solvent environment. The strong interaction between the electrons of the solvent and the cation is analogous to the Lewis acid-base 
interaction; therefore, we refer to the solvation ability of the solvent as Lewis basicity. The method is as follows:

(1) We measured the absorption energies of a dye with $-\mathrm{NH}_{2}$ (4-nitroaniline, $\mathrm{NA}$ ), which corresponds to an acid-base reaction between N-H in NA and a solvent, and the additional Van der Waals attraction.

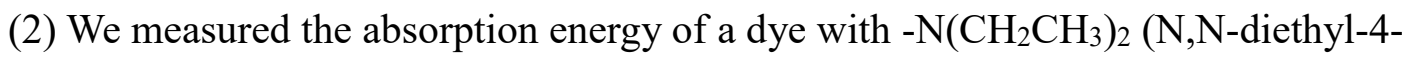
nitroaniline, DA) in the solvent to assess the Van der Waals attraction between DA and solvent.

(3) We fit the absorption energies in (2) to a linear calibration curve of absorption energies between DA and NA in a non-polar solvent to simulate the absorption energy of NA in the absence of the acid-base interaction.

(4) The difference between the absorption energies measured in (1) and (3) produces $\beta$, which reflects the acid-base interaction between the NA and the solvent, while completely removing the Van der Waals effect (Refer to Supplementary Note 2 for the detailed calculation) $)^{38,39}$.

We show that $\beta$ is highly correlated with the solvation structures characterized by Raman spectroscopy — a widely-used tool to assess the lithium-ion-anion-solvent coordination degree — by analysing 14 solvents mixed with $1 \mathrm{M}$ LiTFSI EC/DEC $(\mathrm{EC} / \mathrm{DEC}$ : cosolvent $=$ 1:2) (S1a, S2). The decrease in the $\beta$ of the cosolvent raises AGG and CIP ratios of anions $\left(\mathrm{TFSI}^{-}\right)$, which is the ideal geometry for LHCE (Figure 1b, d, S3 $)^{40}$. Therefore, the battery

CE is more highly correlated with $\beta$ (Pearson's $r:-0.847$ ) than the calculated binding energy 
LMA performance (Figure 1c-d). A widely adopted basicity parameter, the Gutmann donor number, demonstrates lower correlation with the battery CE (Pearson's r: -0.728 ), which supports that $\beta$ is the better basicity parameter (Supplementary Note 1, Figure S1b). This result can be attributed to the $\mathrm{SbCl}_{5}$ Lewis acid being used to calculate the Gutmann donor number that is considerably larger than the lithium-ion. ${ }^{27}$.To the best of our knowledge, such an exclusive effect of $\beta$ on the solvation structure and battery performance is yet to be established.

We propose another solvatochromic parameter, $E_{T}^{N}$, as a descriptor of miscibility because it can directly measure the stabilization energy stemming from the intermolecular attraction between the solvent and mixed probe molecules. Since miscibility is best defined by mixing enthalpies of species in an ensemble, the experimentally characterized intermolecular forces between two dissimilar species better represent the miscibility than the dielectric constant and dipole moment of pure species (Figure 1a, S1c-d, Supplementary Note 3). Although these parameters guide miscibility between dissimilar molecules based on conventional wisdom (i.e., like-dissolves-like), it is clearly limited in precisely determining miscibility. Further, $E_{T}^{N}$ measures the absorption energy of the 2,6-Diphenyl-4-(2,4,6-triphenyl-1-pyridino) phenolate (Reichardt's dye) when it is mixed in the solvent of our interest (Figure 1f) ${ }^{41,42}$. The ground state of the dye becomes more stabilized, and the absorption energy increases when the polar solvent is attracted to the anionic phenolate part of the dye $\mathrm{e}^{43}$. Thus, cosolvents with high $E_{T}^{N}$ warrant the strong attraction to a polar electrolyte, i.e., miscibility within the system (Figure 1a).

We discover the most optimal physicochemical properties for ideal cosolvents essential for the high LMA performance while establishing the correlation of LHCE performance with $\beta$ 
158 and $E_{T}^{N}$ : a low $\beta(<0.2)$ and a medium-high $E_{T}^{N}$. For the non-fluorinated solvents to have 159 such properties, the resonance structure connected to the oxygen atom is introduced to endow 160 solvents with non-solvating, miscible characteristics. We determined three NFNSC - AN, 161 ethoxybenzene, and furan — based on our correlation results and the design rule.

162 Comparison with the non-resonant analogues-methoxycyclohexane (MeCyHx), 163 benzylmethyl ether (BzMe), and tetrahydrofuran (THF) — reveals that molecules with oxygen 164 lone-pair electron resonance structures (Scheme 1) show lower $\beta$ values, higher average CEs, 165 and longer capacity retention compared to those with limited or no resonance structures 166 (Scheme 2) (Figure 2a, c). 


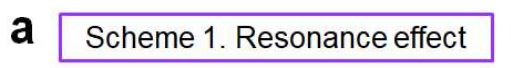

Anisole (AN)

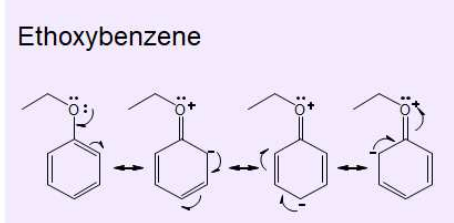

Furan
Coulombic efficiency $(\%)$

HBA basicity
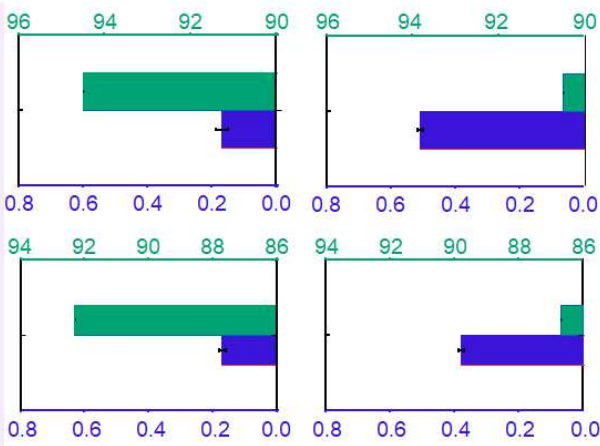

$\begin{array}{llllllllll}96 & 90 & 84 & 78 & 72 & 96 & 90 & 84 & 78 & 72\end{array}$

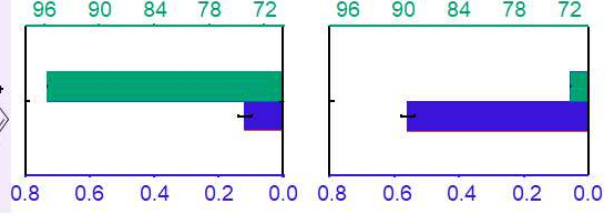

Scheme 2. No resonance effect

Methoxycyclohexane (MeCyHx)

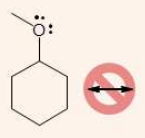

Benzylmethyl Ether (BzMe)

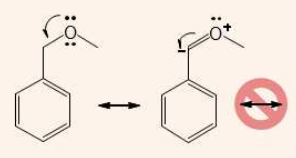

Tetrahydrofuran (THF)

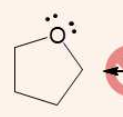

b

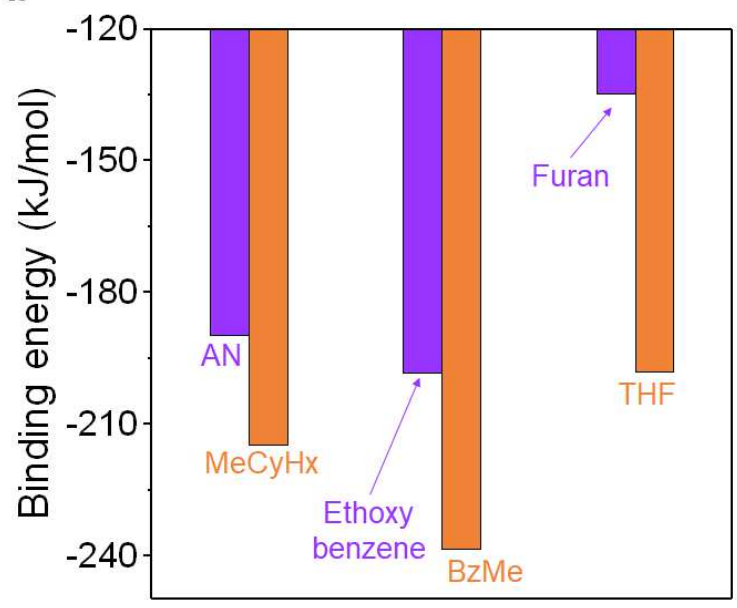

C

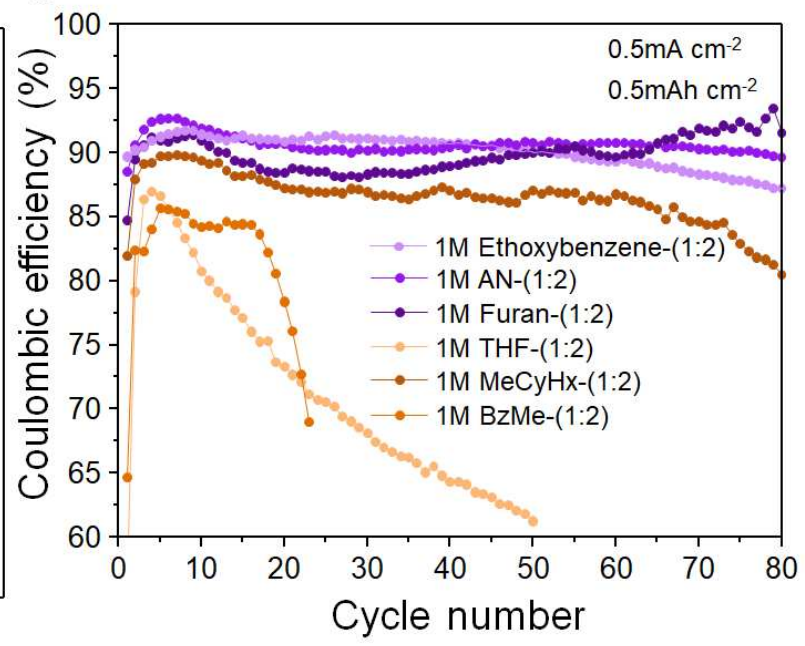

Figure 2. (a) Schematics of the resonance structures of organic compounds and corresponding CE and HBA basicity. (b) Calculated binding energies of lithium-ion with the oxygen atom of each compound. (c) Cycling performance of $\mathrm{Li} \mid \mathrm{Cu}$ cell with different electrolytes at $0.5 \mathrm{~mA} \mathrm{~cm}^{-2}$ to $0.5 \mathrm{mAh} \mathrm{cm}{ }^{-2}$.

DFT calculations also suggest that the resonance can lower the binding energy to lithium-ion

(Figure 2b, Table S1). In the calculation, the binding energies of the lithium-ion to the oxygen atom of AN, furan, and ethoxybenzene are significantly lower than those of analogous molecules despite their similar molecular structures except for the resonance capability. 
Additional calculations were performed on several molecules with phenyl rings and oxygen atoms (Figure S7-8). Comparing oxygen atoms connected directly to the phenyl ring and those blocked by the methylene group, the former atoms exhibit lower binding energies (Supplementary Note 5).

\section{Effect of AN on electrochemical performance of electrolytes}

We tested the electrochemical performance of the 1M LiTFSI EC/DEC (1M EC/DEC) electrolyte, 5M LiTFSI EC/DEC (5M-HCE), and 1M LiTFSI EC/DEC:AN-(1:4) (1M AN(1:4)). The 1:4 ratio of EC/DEC to AN was selected to maintain the local concentration of LiTFSI in EC/DEC as 5M and investigate the diluting effect of AN in the LHCE.

The TFSI- Raman peak shift of 1M AN-(1:4) was identical to that of the 5M-HCE and LiTFSI salt, which confirm their identical solvation structures (Figure 3a). Further, the EC peak of AN-(1:4) demonstrated a similar behaviour as that of 5M-HCE. Further deconvolution of the TFSI $^{-}$peak indicated that the CIP + AGG to SSIP peak ratio increases as the AN ratio increases (Figure S9). Such LHCE solvation structure promotes the anion-derived SEI layer that was observed from the XPS depth profile of the atomic ratio; F and S that comprise the inorganic SEI layer were richer in 1M AN-(1:4) and 5M-HCE compared to 1M EC/DEC (Figure S1011).

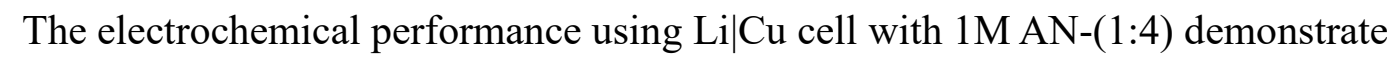
significantly improved cycle life and CE even in high current density and capacity (Figure 3f, S12-13). The superior performance of AN-containing electrolyte compared to 5M-HCE and $1 \mathrm{M} \mathrm{EC/DEC}$ can be attributed to the additional advantages of using non-solvating cosolvents. In addition to $1 \mathrm{M} \mathrm{AN-(1:4)} \mathrm{resembling} \mathrm{the} \mathrm{solvation} \mathrm{structure} \mathrm{of} 5 \mathrm{M}-\mathrm{HCE}$, it has 
a

a Coordinated EC Free AN Coordinated TFSI

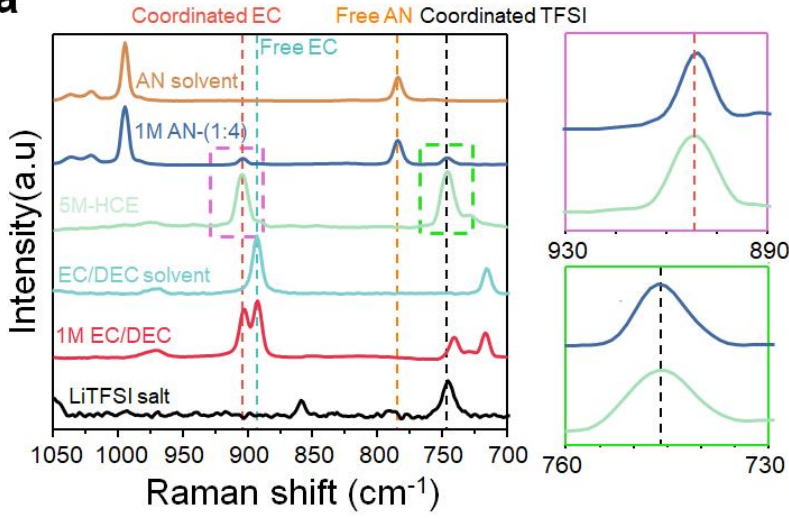

b

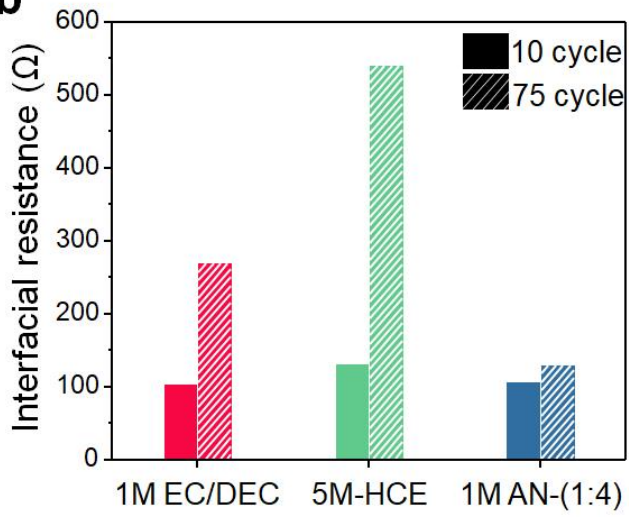

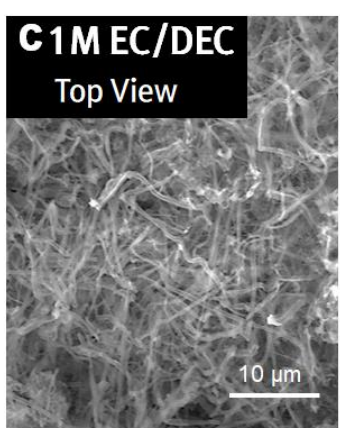

202

The morphology of the lithium deposition of $1 \mathrm{M}$ AN-(1:4) examined using SEM exhibited a
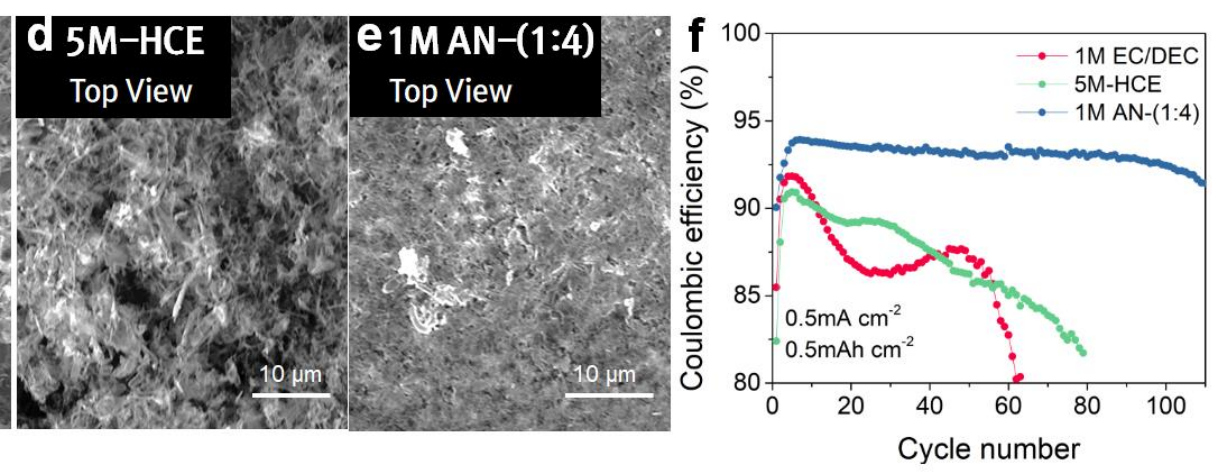

Figure 3. (a) Raman spectra of electrolytes and solvents containing LiTFSI, EC/DEC, and AN. (b) EIS fitted interfacial resistance of different electrolytes at the $10^{\text {th }}$ and $75^{\text {th }}$ cycles. Top-down SEM images of lithium deposition at (c) $1 \mathrm{M} \mathrm{EC/DEC,} \mathrm{(d)} 5 \mathrm{M}-\mathrm{HCE}$, and (e) $1 \mathrm{M} \mathrm{AN-}$ (1:4). (f) Cycling performance of $\mathrm{Li} \mid \mathrm{Cu}$ cell with different electrolytes at $0.5 \mathrm{~mA} \mathrm{~cm}^{-2}$ to 0.5 $\mathrm{mAh} \mathrm{cm}^{-2}$.

$$
\text { significantly more compact lithium deposition morphology than the others (Figure 3c-e, }
$$

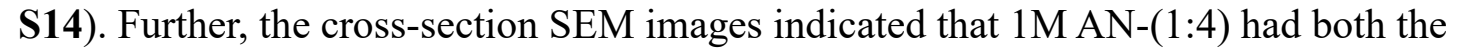

thinnest lithium metal layer with the smoothest surface (Figure S15). According to EIS, the increment in the interfacial resistance from the $10^{\text {th }}$ cycle to the $75^{\text {th }}$ cycle is the smallest in 
214 1M AN-(1:4) (Figure 3b, S16-17). This is further supported by Li 1s XPS depth profile of 215 the charged samples where the lithium metal peak of AN-(1:4) sample is observed to be 216 closer to the surface and online electrochemical mass spectrometry (OEMS) showing less $217 \mathrm{CO}_{2}$ and $\mathrm{C}_{2} \mathrm{H}_{4}$ gas formation in $1 \mathrm{M} \mathrm{AN}-(1: 4)$ (Figure S18-19).

\section{Assessing practicality of NFNSC in LMA}

220

Electrolyte salts and solvents were further optimized to improve cell performance. After varying the salt concentration and volume ratio of AN, the 3M LiFSI DME:AN-(1:2) system shows the highest performance (98.5\% CE for 500 cycles) (Figure 4a and Figure S20). The same electrolyte system was compared with the electrolytes without AN (3M LiFSI DME) and HCE system (9M LiFSI DME). The Li|Cu cells containing 3M LiFSI DME:AN-(1:2) performed the best even when the current density and capacity were raised to $2 \mathrm{~mA} \mathrm{~cm}^{-2}$ and $1 \mathrm{mAh} \mathrm{cm}{ }^{-2}$, respectively (99.1\% CE for 270 cycles) (Figure $4 \mathbf{b}$ ). The full cells with LFP cathodes using thin foil lithium metal $(20 \mu \mathrm{m})$ prove that the capacity retention and ratecapability of AN are superior. 3M LiFSI DME:AN-(1:2) and 3M DME retain $85.4 \%$ and $31.5 \%$ of its capacity at the $300^{\text {th }}$ cycle and $36.9 \%$ and $10.1 \%$ at $20 \mathrm{C}$ compared to that at $0.2 \mathrm{C}$,

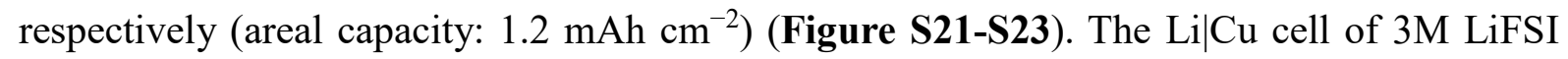
DME:Furan-(1:2) maintains a high CE (99.0\%) for more than 1400 cycles (Figure 4a), and it has the best reported cyclability among LMA LHCE to the best of our knowledge. The superior performance of the furan containing electrolytes was maintained at high current density and capacity $\left(2 \mathrm{~mA} \mathrm{~cm}^{-2}, 1 \mathrm{mAhcm}^{-2}\right)$ as it demonstrated $99.4 \%$ of CE for more than 500 cycles (Figure 4b).

Ionic conductivity significantly increases by more than one order with the addition of NFNSC 
238 NFNSC containing electrolytes with that of 3M LiFSI DME by the EIS analysis, the smaller

SEI layer resistance of the cosolvent containing electrolytes at $1^{\text {st }}$ and $55^{\text {th }}$ deposition and the smaller increase in the resistance confirm that the addition of cosolvents constructs a better anode/electrolyte interphase, which lowers its resistance (Figure 4c, S25).
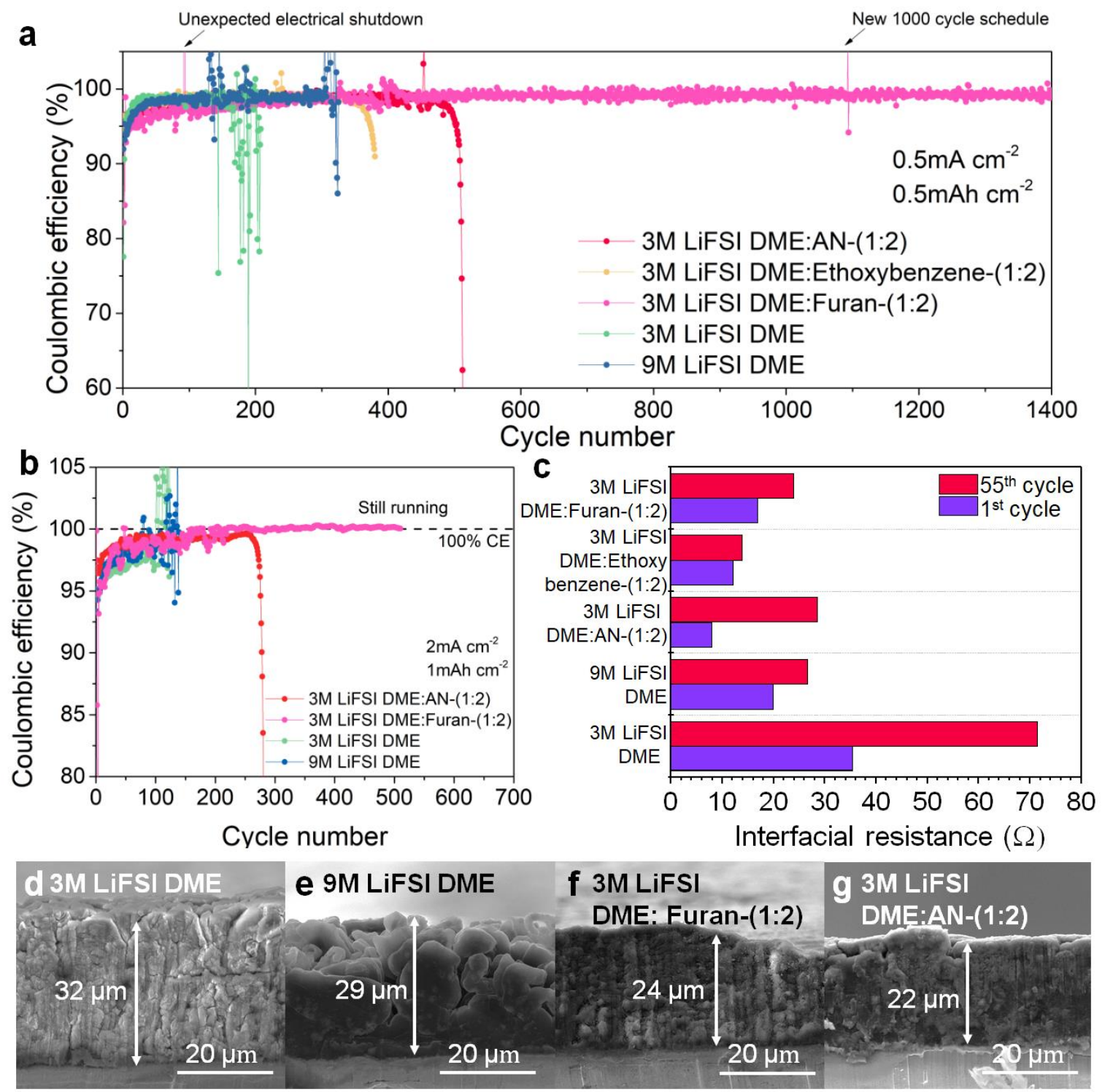

Figure 4. Cycling performance of $\mathrm{Li} \mid \mathrm{Cu}$ cell with different electrolytes at (a) $0.5 \mathrm{~mA} \mathrm{~cm}^{-2}$ to 0.5 $\mathrm{mAh} \mathrm{cm}$, and (b) $2 \mathrm{~mA} \mathrm{~cm}^{-2}$ to $1 \mathrm{mAh} \mathrm{cm}^{-2}$. (c) Interfacial resistance of each electrolyte after $1^{\text {st }}$ and $55^{\text {th }}$ cycle obtained from the EIS. Cross-section SEM image of (d) 3M LiFSI DME, (e) 9M LiFSI DME, (f) 3M LiFSI DME:Furan-(1:2), and (g) 3M LiFSI DME:AN-(1:2), which each 
having depths of $32 \mu \mathrm{m}, 29 \mu \mathrm{m}, 24 \mu \mathrm{m}$, and $22 \mu \mathrm{m}$, respectively.

Cross-section SEM data demonstrated that the deposited lithium metals in both 3M LiFSI

DME:AN-(1:2) and 3M LiFSI DME:Furan-(1:2) were more compact and thinner compared to those in 3M and 9M LiFSI DME (Figure 4d-g, S27). Ethoxybenzene electrolyte (3M LiFSI DME:ethoxybenzene-(1:2)) renders relatively thicker initial deposition, and it may be responsible for the lowest cyclability among NFNSC-containing electrolytes (Figure S25-26).

\section{Quantification of cosolvents in electrolytes}

AN and furan-containing electrolytes demonstrated longer cycling than the reported cyclability of DFB (350 cycles) and FB (500 cycles), which contain fluorine and induce the LiF formation on $\mathrm{LMA}^{14,15}$. We attribute the long cyclabilities of our electrolytes to the high-energy position of its LUMO. A LUMO level of a solvent is one of the dominating factors that determines the performance of an electrolyte especially after extended cycling. A high LUMO level of the solvent guarantees its high stability under reductive condition ${ }^{44,45}$. Our quantum chemical calculations indicate that the LUMO level of $\mathrm{AN}$ is $-0.543 \mathrm{eV}$ and that of furan is $-0.195 \mathrm{eV}$; however, that of DFB is $-0.950 \mathrm{eV}$, which is substantially lower (Figure 5a). The addition of electronegative fluorine lowers the LUMO level, which aggravates the reduction and decomposition of a cosolvent more preferably at the lithium anode interphases ${ }^{14,15,20}$. Such a facile decomposition of a cosolvent not only leads to an electrolyte dry-up but may also decrease partial concentration, which can result in the overall composition deviation from the initially designed electrolyte with cycling ${ }^{21}$. We found that a decrease in the relative composition of cosolvents can decrease the CE after extended cycling and induce battery failure. 

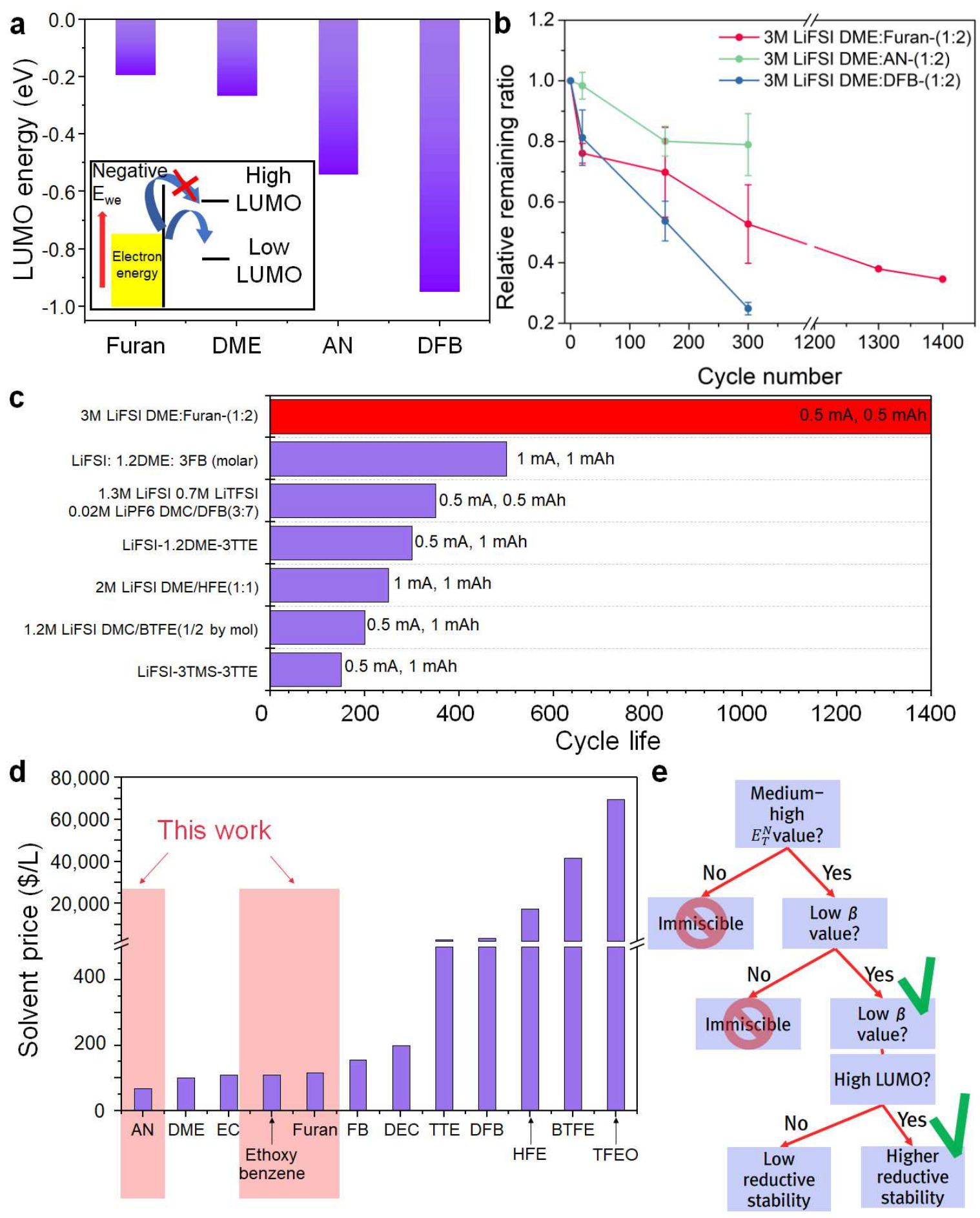

272 Figure 5. (a) Calculated LUMO energy of different solvents (Furan, DME, FB, and DFB). (b) 273 Relative remaining ratio of each solvent after cycling the Li|Cu cells at $0.5 \mathrm{~mA} \mathrm{~cm}^{-2}$ to $0.5 \mathrm{mAh}$ $274 \mathrm{~cm}^{-2}$ for the designated cycle number. (c) Comparison of $\mathrm{Li} \mid \mathrm{Cu}$ cell performance with various 
cosolvents reported in previous studies. Their areal current density and areal capacity values are indicated. (d) Comparison of the prices of (co)solvents commonly utilized in the LMA electrolyte system. (e) A logic scheme for choosing an appropriate NFNSC.

Linear sweep voltammetry (LSV) of 1M DFB-(1:2) during initial charging exhibited additional reduction peaks at a higher voltage (Figure S28), which suggest lower cathodic stability caused by a low LUMO. In addition, ${ }^{1} \mathrm{H}-\mathrm{NMR}$ spectroscopy was performed for electrolytes extracted from the $\mathrm{Li} \mid \mathrm{Cu}$ cells after cycling to quantify the concentration of the remaining cosolvents (Supplementary Note 7 for the specific method). The remaining quantity of furan and AN at the extended cycles is higher than that of DFB with cycles (Figure 5b). About $35 \%$ of furan remained even after 1400 cycles, which is higher than the remaining ratios of DFB at 300 cycles. Our measurement does not detect the noticeable degradation of DME because of its higher LUMO level compared to that of other cosolvents in this study; this suggests that the degradation of cosolvents is responsible for the cycle capability (Figure S29a).

Overcoming the cycle capability of LMA longer than 500 cycles with a CE higher than $99.0 \%$ has not been achieved before by any other conventional studies that rely on expensive fluorinated cosolvents to the best of our knowledge $\mathrm{e}^{1,2,6,14,15,46}$. Therefore, our design rule of the cosolvent unlocks a route toward the LMA battery with an exceptionally long cycle life (Figure 5c). Along with the high $\mathrm{CE}$ and long cyclability, the cost of electrolytes has a major criteria in assessing their practicality. The solvents investigated in this work are compared with other cosolvents by their prices (Figure 5d). Many cosolvents that have been reported to date are considerably more expensive, and this increases the battery production cost. Common, fluorine-free organic compounds such as $\mathrm{AN}$, furan, and ethoxybenzene are considerably inexpensive and comparable to common solvents such as EC and DME in terms of cost. 
Thus, we propose a logic scheme for selecting an appropriate non-solvating cosolvent for a

300

301

302

303

304

305

306

307

308

309

310

311

312

313

314

315

316

317

318

319

high-performance LMA electrolyte. The cosolvent should have a medium-high $E_{T}^{N}$ to be miscible with polar electrolytes, a low $\beta$ value $(<0.2)$ to induce anion-coordinated solvation structure, and a high LUMO to remain stable in a highly reductive condition (Figure 5e).

\section{Conclusion}

In this study, we proposed critical parameters for optimal non-solvating cosolvents and correlated their effects with the electrochemical performance of various electrolytes. These insights enable us to replace the conventional FNSC with NFNSC which show superior cathodic stability confirmed by the long cyclability of 3M LiFSI DME:furan-(1:2) electrolyte. This work represents a significant advancement in designing non-solvating cosolvents by providing new means to tune the solvation ability of a molecule without aggravating the reductive stability. Also, the design rule proposed here can be useful in determining solvation ability and predicting the performance of newly-tested solvents in lithium metal battery electrolyte. Overall, our proposal opens a new horizon for designing electrolytes for high performance LMA.

\section{Methods/Experimental Section}

\section{Materials and Electrolyte Preparation}

LiTFSI (99.95\%, trace metal basis), ethoxybenzene (99\%), fluorobenzene (99\%), benzylmethyl ether (98\%), dibenzyl ether (>98\%), diphenyl ether (>99\%), 1,2dimethoxyethane (99.5\%, anhydrous, inhibitor free), ethylene carbonate (99\%, anhydrous), diethyl carbonate (>99\%, anhydrous), dibutyl ether (99.30\%, anhydrous), 1,3-dioxolane 
320

321

322

323

324

325

326

327

328

329

330

331

332

333

334

335

336

337

338

339

340

341

(99.80\%, anhydrous, contains $~ 75$ ppm BHT as inhibitor, 99.8\%), furan (>99\%), 4-nitroaniline (>99\%) were purchased from Sigma-Aldrich. LiFSI (>98\%), 1,2-difluorobenzene (>98\%), methoxycyclohexane (>98\%), diisopropyl ether (>99\%) were purchased from Tokyo Chemical Industry Co., Ltd. AN (99\%), methyl tert-butyl ether (HPLC grade, anhydrous), tetrahydrofuran (99.8+\%, anhydrous), 1,4-dioxane (anhydrous, 99.8\%, stab. with 1-3ppm BHT), LiCl (ultra dry, 99.995\%) were purchased from Alfa Aesar. LiFSI (>99.97\%) was purchased from Chunbo. Deuterated dimethyl sulfoxide (DMSO-d ${ }^{6},>99.8 \%$ ) NMR solvent was purchased from Deutero. N,N-Diethyl-4-nitroaniline (98\%) was purchased from CombiBlocks. All solvents except EC were stored under a $4 \AA$ molecular sieve (Alfa Aesar) for at least two days and all electrolytes were prepared in an Ar-filled glove box. As the volumetric ratio of EC/DEC was fixed to 1:1 for all the experiments, we do not explicitly indicate the ratio for this mixture in the main article.

\section{Calculating Solvatochromic $\beta$ parameter}

The $\beta$ parameter was calculated based on the method proposed by Kamlet et al. ${ }^{39}$ The measurement was performed using a Shimadzu UV-2600 spectrophotometer. The spectral range was $300-500 \mathrm{~nm}$ with a resolution of $0.1 \mathrm{~nm}$. The final concentration of the dye was 1.0 $\times 10^{-4} \mathrm{M}$ and each solvent was measured at least three times.

\section{Electrochemical Testing}

2032-type coin cells were assembled in an Ar-filled glove box. A Celgard 2320 microporous membrane separator and $80 \mu \mathrm{L}$ of electrolyte were used for integrating each cell. All cycling experiments were performed at $25^{\circ} \mathrm{C}$ using a WBCS3000L battery cycler (WonATech). Prior to any cycling experiment, the $\mathrm{Li} \mid \mathrm{Cu}$ cells were rested for $3 \mathrm{~h}$ at the $\mathrm{OCV}$ and then cycled at 
the predetermined areal current and capacity. The cut-off voltage for each cycle was set to $1 \mathrm{~V}$ (vs. $\mathrm{Li} / \mathrm{Li}^{+}$). The EIS and cyclic voltammetry (CV) were performed using VSP-300 (BioLogic) in $\mathrm{Li} \mid \mathrm{Cu}$ asymmetric cells. Both were measured at room temperature and rested for 30 seconds at OCV before the measurement. The EIS measurements were conducted after the discharging cycle. The frequency range was from $100 \mathrm{kHz}$ to $0.1 \mathrm{~Hz}$ with a $10 \mathrm{mV}$ amplitude.

\section{Characterization}

Cells were disassembled in an Ar-filled glove box. The electrodes were washed with the DEC or DME solvent and dried under vacuum.

SEM (MIRA3 XMH, TESCAN) was used for morphology characterization. Loaded SEM samples were directly placed into the chamber to minimize air contact. The accelerating voltage was set to $15 \mathrm{kV}$.

The chemical compositions and bonding characteristics of the SEI layers were analysed using XPS (Thermo VG Scientific (Sigma Probe, Mg Ka source)) and all peaks were fitted according to the $\mathrm{C}-\mathrm{C}$ bond $284.8 \mathrm{eV}$ reference peak. For the survey scan, a $1.0 \mathrm{eV}$ step size was used, and a $0.1 \mathrm{eV}$ step size was used for the high-resolution scans for all elements.

The coordination structure of various electrolytes was studied by Raman spectroscopy using an inVia Raman Microscope (Renishaw) with an exciting laser of $514 \mathrm{~nm}$. The peak deconvolution of the obtained spectra was performed with a Gaussian function. For each electrolyte, at least three spectra were reproduced on different days. The deconvoluted peak ratios are the average values and the error bar is the $95 \%$ confidential interval. 
For the co-solvent and salt quantification, $\mathrm{Li} \mid \mathrm{Cu}$ coin cells containing $40 \mu \mathrm{L}$ of electrolytes were cycled for specific cycles at $0.5 \mathrm{~mA} \mathrm{~cm}^{-2}$ to $0.5 \mathrm{mAh} \mathrm{cm}^{-2}$. All coin cell parts were disassembled and placed in a PTFE vial with $1 \mathrm{~mL}$ of DMSO-d $6.20 \mu \mathrm{L}$ of EC was used as an internal standard for ${ }^{1} \mathrm{H}$ NMR. The vials were shaken for $5 \mathrm{~min}^{47}$. The ${ }^{1} \mathrm{H}$ NMR was performed using $500 \mathrm{MHz}$ NMR (Varian 500). Peak deconvolution was performed with Mestrenova software using the Lorentzian fit method. This process was repeated at least three times for each sample.

\section{Computational Details}

Quantum chemical calculations were performed with the Gaussian 16, Revision A.03 program using the density functional theory (DFT) B3LYP functional using Grimme Dispersion (D3BJ) and ultrafine integration ${ }^{48,49}$. Structure optimization was performed with the cc-pVTZ basis set and a frequency calculation was performed to verify the absence of imaginary frequencies. Single-point energy calculations were performed with the aug-cc-pVQZ basis set. From the obtained structures, the binding energies and LUMO energy levels were obtained.

\section{Acknowledgements}

This work was partly supported by the National Research Foundation of Korea (NRF) grants funded by the Korean government (MSIT) (no. NRF-2021R1C1C1013953),

Industrial Strategic Technology Development Program-Alchemist Project (20012390, 4D Molecular-Nano-Addressable Lithographic Self-Assembly (4D MONALISA) funded By the 
382

383

384

385

386

387 Author Contributions

388

389

390

391

392

393

394

395

396

397

398

399

400

401

Development (ADD, UC190025RD). manuscript.

\section{Reference}

Ministry of Trade, Industry \& Energy (MOTIE, Korea), and the Agency for Defense

We express thanks to the staff and crew of the Research Institute of Advanced Materials (RIAM) and the Institute of Applied Physics of Seoul National University.

J.M., D.K., and J.L. conceived the project and designed the experiments. J.M., D.K. did the Li|Cu electrochemical testing and NMR spectroscopy. L.B. did the computational calculations. M.S. and D.W. did the OEMS experiments. J.Y. and J.C. conducted the full-cell experiments. J.Y. did the SEM and XPS characterizations. D.K. did the Raman experiment. J.Y. and D.K. co-wrote the manuscript. All the authors discussed the results and commented on the

1. Chen, S. et al. High-Voltage Lithium-Metal Batteries Enabled by Localized High-Concentration Electrolytes. Adv. Mater. 30, 1706102 (2018).

2. Ren, X. et al. Localized High-Concentration Sulfone Electrolytes for High-Efficiency LithiumMetal Batteries. Chem 4, 1877-1892 (2018).

3. Chen, J. et al. Highly Reversible Lithium-Metal Anode and Lithium-Sulfur Batteries Enabled by an Intrinsic Safe Electrolyte. ACS Appl. Mater. Interfaces 11, 33419-33427 (2019). 
402

4. Takada, K., Yamada, Y. \& Yamada, A. Optimized Nonflammable Concentrated Electrolytes by Introducing a Low-Dielectric Diluent. ACS Appl. Mater. Interfaces 11, 35770-35776 (2019).

5. Han, S. Structure and dynamics in the lithium solvation shell of nonaqueous electrolytes. Sci. Rep. 9, 5555 (2019).

6. Ren, X. et al. Enabling High-Voltage Lithium-Metal Batteries under Practical Conditions. Joule 3, 1662-1676 (2019).

7. Xia, J. et al. Fluorinated electrolyte for $4.5 \mathrm{~V}$ Li(Ni0.4Mn0.4Co0.2)O2/graphite Li-ion cells. J. Power Sources 307, 340-350 (2016).

8. Suo, L. et al. Fluorine-donating electrolytes enable highly reversible 5-V-class Li metal batteries. Proc. Natl. Acad. Sci. 115, 1156-1161 (2018).

9. Lin, D., Liu, Y. \& Cui, Y. Reviving the lithium metal anode for high-energy batteries. Nat. Nanotechnol. 12, 194-206 (2017).

10. Cheng, X.-B., Zhang, R., Zhao, C.-Z. \& Zhang, Q. Toward Safe Lithium Metal Anode in Rechargeable Batteries: A Review. Chem. Rev. 117, 10403-10473 (2017).

11. Wang, H., Liu, Y., Li, Y. \& Cui, Y. Lithium Metal Anode Materials Design: Interphase and Host. Electrochem. Energy Rev. 2, 509-517 (2019).

12. Li, T., Zhang, X.-Q., Shi, P. \& Zhang, Q. Fluorinated Solid-Electrolyte Interphase in High-Voltage Lithium Metal Batteries. Joule 3, 2647-2661 (2019).

13. He, M., Guo, R., Hobold, G. M., Gao, H. \& Gallant, B. M. The intrinsic behavior of lithium fluoride 
14. Yoo, D., Yang, S., Kim, K. J. \& Choi, J. W. Fluorinated Aromatic Diluent for High-Performance Lithium Metal Batteries. Angew. Chem. Int. Ed. 59, 14869-14876 (2020).

15. Jiang, Z. et al. Fluorobenzene, A Low-Density, Economical, and Bifunctional Hydrocarbon Cosolvent for Practical Lithium Metal Batteries. Adv. Funct. Mater. 31, 2005991 (2021).

16. Ding, J.-F. et al. Non-Solvating and Low-Dielectricity Cosolvent for Anion-Derived Solid doi:10.1002/anie.202101627.

17. Su, C., He, M., Amine, R. \& Amine, K. A Selection Rule for Hydrofluoroether Electrolyte Cosolvent:

18. Abdou, H. E., Mohamed, A. A., López-de-Luzuriaga, J. M., Monge, M. \& Fackler, J. P. Fine-Tuning

19. Zhang, M., Guo, X., Zhang, S. \& Hou, J. Synergistic Effect of Fluorination on Molecular Energy

20. Cao, X. et al. Monolithic solid-electrolyte interphases formed in fluorinated orthoformate-based electrolytes minimize Li depletion and pulverization. Nat. Energy 4, 796-805 (2019). 
22. Wang, A., Kadam, S., Li, H., Shi, S. \& Qi, Y. Review on modeling of the anode solid electrolyte interphase (SEI) for lithium-ion batteries. Npj Comput. Mater. 4, 15 (2018).

23. Niu, C. et al. Balancing interfacial reactions to achieve long cycle life in high-energy lithium metal batteries. Nat. Energy 6, 723-732 (2021).

24. Niu, C. et al. High-energy lithium metal pouch cells with limited anode swelling and long stable cycles. Nat. Energy 4, 551-559 (2019).

25. Subramanian, M. A. A 'Greener' Synthetic Route for Fluoroaromatics via Copper (II) Fluoride.

26. Kyzer, J. L. \& Martens, M. Metabolism and Toxicity of Fluorine Compounds. Chem. Res. Toxicol. Science 297, 1665-1665 (2002).

27. Yamada, Y. \& Yamada, A. Review-Superconcentrated Electrolytes for Lithium Batteries. J.

28. Yamada, Y., Wang, J., Ko, S., Watanabe, E. \& Yamada, A. Advances and issues in developing saltconcentrated battery electrolytes. Nat. Energy 4, 269-280 (2019).

29. Cao, X., Jia, H., Xu, W. \& Zhang, J.-G. Review-Localized High-Concentration Electrolytes for Lithium Batteries. J. Electrochem. Soc. 168, 010522 (2021). Electrolyte**. Angew. Chem. Int. Ed. 60, 4090-4097 (2021). 
31. Chernyak, Y. Dielectric Constant, Dipole Moment, and Solubility Parameters of Some Cyclic Acid Esters. J. Chem. Eng. Data 51, 416-418 (2006).

32. Reichardt, C. et al. Solute/solvent interactions and their empirical determination by means of solvatochromic dyes. Pure Appl. Chem. 65, 2593-2601 (1993).

33. Bezabh, H. K. et al. Roles of film-forming additives in diluted and concentrated electrolytes for lithium metal batteries: A density functional theory-based approach. Electrochem. Commun.

34. Zhuang, B., Ramanauskaite, G., Koa, Z. Y. \& Wang, Z.-G. Like dissolves like: A first-principles 113, 106685 (2020).

35. Obukata, T., Yamada, Y. \& Yamada, A. Controlling the coordination states of electrolyte solutions for reversible lithium metal plating/stripping. 1.

36. Aldeghi, M., Heifetz, A., Bodkin, M. J., Knapp, S. \& Biggin, P. C. Accurate calculation of the absolute free energy of binding for drug molecules. Chem. Sci. 7, 207-218 (2016).

37. Chaban, V. Competitive solvation of (bis)(trifluoromethanesulfonyl)imide anion by acetonitrile and water. Chem. Phys. Lett. 613, 90-94 (2014).

38. Nigam, S. \& Rutan, S. Principles and Applications of Solvatochromism. Appl. Spectrosc. 55, 362A-370A (2001). 
40. Yamada, Y. et al. Unusual Stability of Acetonitrile-Based Superconcentrated Electrolytes for FastCharging Lithium-Ion Batteries. J. Am. Chem. Soc. 136, 5039-5046 (2014).

41. Nigam, S. \& Rutan, S. Principles and Applications of Solvatochromism. Appl. Spectrosc. 55, 362A-370A (2001).

42. Reichardt, C. Solvatochromic Dyes as Solvent Polarity Indicators. Chem. Rev. 94, 2319-2358 (1994).

43. Dimroth, K., Reichardt, C., Siepmann, T. \& Bohlmann, F. Über Pyridinium-N-phenol-betaine und (2014).

45. Borodin, O. Challenges with prediction of battery electrolyte electrochemical stability window and guiding the electrode - electrolyte stabilization. Curr. Opin. Electrochem. 13, 86-93 (2019).

46. Lin, S. \& Zhao, J. Functional Electrolyte of Fluorinated Ether and Ester for Stabilizing Both $4.5 \mathrm{~V}$ $\mathrm{LiCoO}_{2}$ Cathode and Lithium Metal Anode. ACS Appl. Mater. Interfaces 12, 8316-8323 (2020).

47. Louli, A. J. et al. Diagnosing and correcting anode-free cell failure via electrolyte and morphological analysis. Nat. Energy 5, 693-702 (2020). 

98, 5648-5652 (1993).

498 49. Parr, R. G. \& Yang, W. Density-functional theory of atoms and molecules. (Oxford Univ. Press 499 [u.a.], 1994).

500 


\section{Supplementary Files}

This is a list of supplementary files associated with this preprint. Click to download.

- SupplementaryMaterialsfinal1028.pdf 